Document downloaded from:

http://hdl.handle.net/10251/99677

This paper must be cited as:

Albanese, A.; Bonet Solves, JA.; Ricker, WJ. (2016). The Cesàro operator in growth Banach spaces of analytic functions. Integral Equations and Operator Theory. 86(1):97-112. doi:10.1007/s00020-016-2316-z

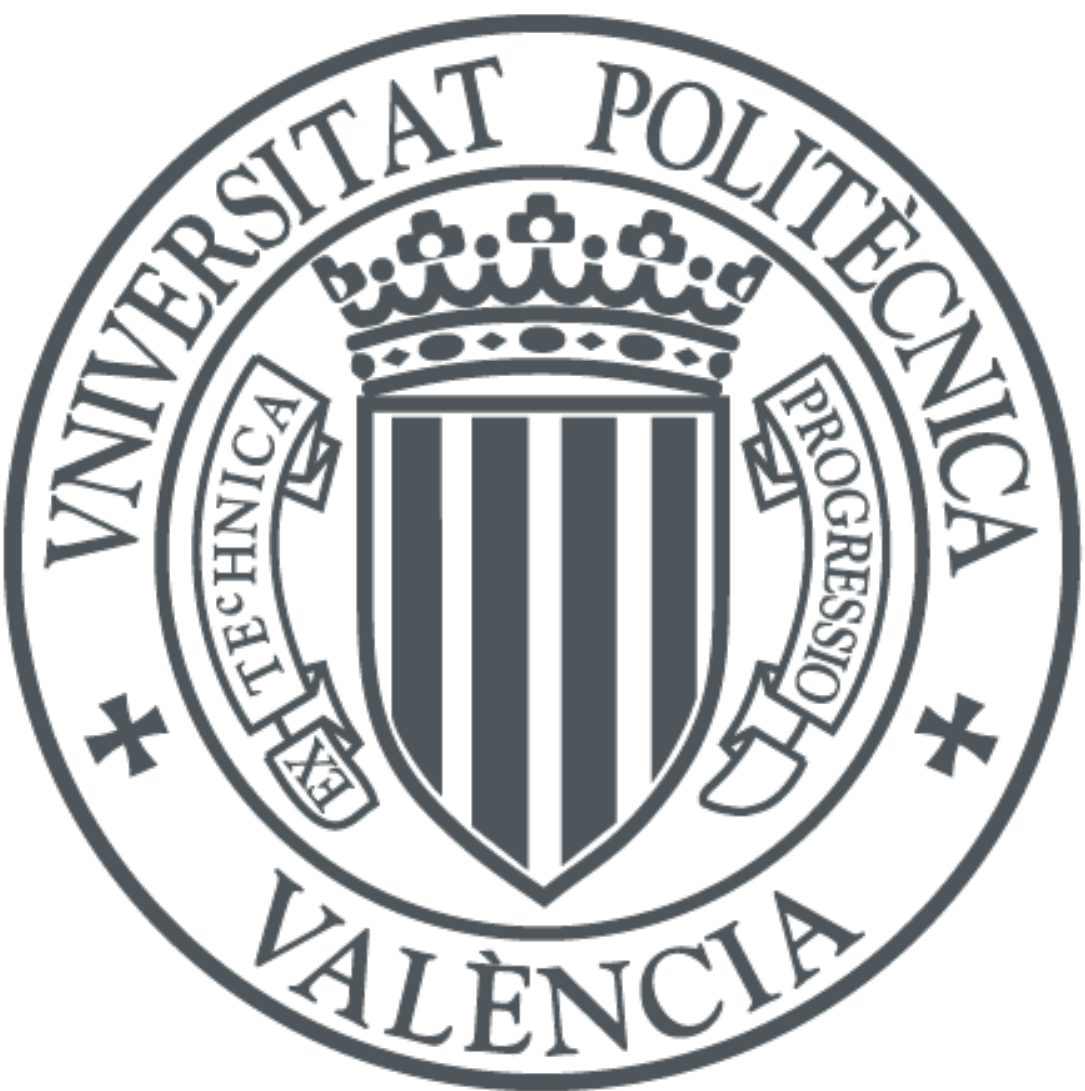

The final publication is available at

http://doi.org/10.1007/s00020-016-2316-z

Copyright Springer-Verlag

Additional Information 


\title{
THE CESȦRO OPERATOR IN GROWTH BANACH SPACES OF ANALYTIC FUNCTIONS
}

\author{
ANGELA A. ALBANESE, JOSÉ BONET, WERNER J. RICKER
}

\begin{abstract}
The Cesàro operator $\mathrm{C}$, when acting in the classical growth Banach spaces $A^{-\gamma}$ and $A_{0}^{-\gamma}$, for $\gamma>0$, of analytic functions on $\mathbb{D}$, is investigated. Based on a detailed knowledge of their spectra (due to A. Aleman and A.-M. Persson) we are able to determine the norms of these operators precisely. It is then possible to characterize the mean ergodic and related properties of $\mathrm{C}$ acting in these spaces. In addition, we determine the largest Banach space of analytic functions on $\mathbb{D}$ which C maps into $A^{-\gamma}$ (resp. into $A_{0}^{-\gamma}$ ); this optimal domain space always contains $A^{-\gamma}$ (resp. $A_{0}^{-\gamma}$ ) as a proper subspace.
\end{abstract}

\section{INTRODUCTION}

Let $H(\mathbb{D})$ denote the Fréchet space of all holomorphic functions $f: \mathbb{D} \rightarrow \mathbb{C}$ equipped with the topology of uniform convergence on the compact subsets of the open unit disc $\mathbb{D}$. The classical Cesàro operator $C$ is given by

$$
f \mapsto \mathrm{C}(f): z \mapsto \frac{1}{z} \int_{0}^{z} \frac{f(\zeta)}{1-\zeta} d \zeta, \quad z \in \mathbb{D} \backslash\{0\}, \quad \text { and } \quad \mathrm{C}(f)(0)=f(0),
$$

for $f \in H(\mathbb{D})$. It is a Fréchet space isomorphism of $H(\mathbb{D})$ onto itself. In terms of the Taylor coefficients $\widehat{f}(n):=\frac{f^{(n)}(0)}{n !}$, for $n \in \mathbb{N}_{0}$, of functions $f(z)=\sum_{n=0}^{\infty} \widehat{f}(n) z^{n} \in H(\mathbb{D})$ one has the description

$$
\mathrm{C}(f)(z)=\sum_{n=0}^{\infty}\left(\frac{1}{n+1} \sum_{k=0}^{n} \widehat{f}(k)\right) z^{n}, \quad z \in \mathbb{D} .
$$

A vector space $X \subseteq H(\mathbb{D})$ is called a Banach space of analytic functions on $\mathbb{D}$ if it is a Banach space relative to a norm for which the natural inclusion of $X$ into $H(\mathbb{D})$ is continuous. Since evaluation at points of $\mathbb{D}$ are continuous linear functionals on $H(\mathbb{D})$, this is equivalent to each evaluation functional $\delta_{z}: f \mapsto f(z)$ at a point $z \in \mathbb{D}$ being an element of the dual Banach space $X^{*}$ of $X$. In this case, the Cesàro operator $C$ is said to act in $X$ if it maps $X$ into itself, i.e. $\mathrm{C}(X) \subseteq X$; by the closed graph theorem $\mathrm{C}$ is necessarily continuous. It is known that $\mathrm{C}$ acts in many classical Banach spaces of

Key words and phrases. Cesàro operator, growth Banach space of analytic functions, mean ergodic operator, optimal domain.

Mathematics Subject Classification 2010: Primary 47B38; Secondary 46E15, 47A10, 47A16, 47A35. 
analytic functions on $\mathbb{D}$; for instance, the Hardy spaces $H^{p}(\mathbb{D}), 1 \leq p<$ $\infty$, the Bergman and the Dirichlet spaces, etc. (see $[5,6,8,9,31]$ and the references therein). It also acts in certain weighted Banach spaces of analytic functions on $\mathbb{D}$, such as weighted Hardy spaces $H^{p}(w), 1<p<\infty$, and others, $[4,7,15,28]$. On the other hand, $\mathrm{C}$ fails to act in $H^{\infty}(\mathbb{D})$ since $\mathrm{C}(\mathbf{1})(z)=(1 / z) \log (1 /(1-z))$, for $z \in \mathbb{D}$; see [16] for an investigation of $\mathrm{C}$ on $H^{\infty}(\mathbb{D})$. Once $\mathrm{C}$ is known to act in $X$, it is desirable to identify its spectrum $\sigma(\mathrm{C})$ and its point spectrum $\sigma_{p t}(\mathrm{C})$ as these often influence various operator theoretic properties of $\mathrm{C}$ (e.g. power boundedness, mean ergodicity, linear dynamics, decomposability, etc).

The aim of this note is to investigate various properties of the Cesàro operator $\mathrm{C}$ related to its action in the classical growth Banach space $A^{-\gamma}$, for each $\gamma>0$, consisting of those analytic functions on $\mathbb{D}$ specified by

$$
A^{-\gamma}:=\left\{f \in H(\mathbb{D}):\|f\|_{-\gamma}:=\sup _{z \in \mathbb{D}}(1-|z|)^{\gamma}|f(z)|<\infty\right\}
$$

and its (proper) closed subspace

$$
A_{0}^{-\gamma}:=\left\{f \in H(\mathbb{D}): \lim _{|z| \rightarrow 1^{-}}(1-|z|)^{\gamma}|f(z)|=0\right\},
$$

equipped with the norm

$$
\|f\|_{-\gamma}:=\sup _{z \in \mathbb{D}}(1-|z|)^{\gamma}|f(z)|, \quad f \in A^{-\gamma} .
$$

Since $(1-|z|)^{\gamma} \leq\left(1-|z|^{2}\right)^{\gamma} \leq 2^{\gamma}(1-|z|)^{\gamma}$, for $z \in \mathbb{D}$, these are the same spaces (with an equivalent norm) as those treated in [21, Ch.4].

The space $A_{0}^{-\gamma}$ coincides with the closure of the polynomials in $A^{-\gamma},[30$, Lemma 3], and point evaluations on $\mathbb{D}$ belong to both $\left(A_{0}^{-\gamma}\right)^{*}$ and $\left(A^{-\gamma}\right)^{*}$, [30, Lemma 1]. Moreover, the bidual $\left(A_{0}^{-\gamma}\right)^{* *}=A^{-\gamma}$ for all $\gamma>0$, [29], [30, Theorem 2]. Shields and Williams, [30], proved that $A^{-\gamma}$ is isomorphic to $\ell^{\infty}$ and that $A_{0}^{-\gamma}$ is isomorphic to $c_{0}$, for each $\gamma>0$; see also Theorem 1.1 in the paper by Lusky, [25]. The growth Banach spaces $A^{-\gamma}$ and $A_{0}^{-\gamma}$ play an important role in connection with the interpolation and sampling of holomorphic functions, [21, Ch. 4 \& 5]. They are particular examples of weighted Banach spaces $H_{v}^{\infty}$ and $H_{v}^{0}$ of holomorphic functions on $\mathbb{D}$, which have been investigated by several authors since the work of Shields and Williams, [30]; see, for example, [11, 12, 13, 25] and the references therein. Observe, for each pair $0<\mu_{1}<\mu_{2}$, that $A^{-\mu_{1}} \subseteq A_{0}^{-\mu_{2}}$.

Recently, Aleman and Persson have made an extensive investigation of various properties (including the spectrum) of generalized Cesàro operators acting in a large class of Banach spaces of analytic functions on $\mathbb{D}$, [7], [28]. In particular, their results apply to the classical Cesàro operator $C$ given by (1.1) when acting in the growth spaces $A^{-\gamma}, A_{0}^{-\gamma}$ for $\gamma>0$. For this setting our additional results will complement and extend their work. For instance, they show that $\mathrm{C}$ acts in both $A^{-\gamma}$ (where we denote it by $\mathrm{C}_{\gamma}$ ) and in $A_{0}^{-\gamma}$ (where we denote it by $\mathrm{C}_{\gamma, 0}$ ). This follows from Theorem 4.1 of 
[7] with $g(z)=-\log (1-z)$, for $z \in \mathbb{D}$, but no quantitative estimates of the operator norms $\left\|\mathrm{C}_{\gamma}\right\|$ and $\left\|\mathrm{C}_{\gamma, 0}\right\|$ are given. We show in Theorem 2.3, that

$\left\|C_{\gamma}^{n}\right\|=\left\|C_{\gamma, 0}^{n}\right\|=1$, for $\gamma \geq 1$, and $\left\|C_{\gamma}^{n}\right\|=\left\|C_{\gamma, 0}^{n}\right\|=1 / \gamma^{n}$, for $0<\gamma<1$,

for each $n \in \mathbb{N}$. Crucial for the proof of Theorem 2.3 is knowing the precise nature of the spectra $\sigma\left(\mathbf{C}_{\gamma}\right)$ and $\sigma\left(\mathbf{C}_{\gamma, 0}\right)$, which have been completely determined by Aleman and Persson, [7, 28]. This knowledge of the spectra, combined with (1.2), makes it possible to determine the mean ergodic properties of $\mathrm{C}_{\gamma}$ and $\mathrm{C}_{\gamma, 0}$. Namely, it turns out that both $\mathrm{C}_{\gamma}$ and $\mathrm{C}_{\gamma, 0}$ fail to be mean ergodic when $0<\gamma \leq 1$ but, both operators are (even uniformly) mean ergodic for all $\gamma>1$; see Theorem 2.5 below. Somewhat surprisingly, the ranges $\operatorname{Im}\left(\mathrm{I}-\mathrm{C}_{\gamma}\right):=\left\{\left(\mathrm{I}-\mathrm{C}_{\gamma}\right)(\mathrm{f}): \mathrm{f} \in \mathrm{A}^{-\gamma}\right\}$, resp. $\operatorname{Im}\left(\mathrm{I}-\mathrm{C}_{\gamma, 0}\right)$, are closed in $A^{-\gamma}$, resp. $A_{0}^{-\gamma}$, for all positive $\gamma \neq 1$ but, not for $\gamma=1$; see Theorem 2.5. None of the Cesàro operators $C_{\gamma}, C_{\gamma, 0}$, for $\gamma>0$, are supercyclic and hence, they also fail to be hypercyclic (cf. Proposition 2.7).

The final section is devoted to determining the optimal domain (a concept introduced in [15]) for both of the operators $C_{\gamma}$ and $C_{\gamma, 0}$ for all $\gamma>0$. That is, we are able to identify the largest Banach space of analytic functions on $\mathbb{D}$, denoted by $\left[\mathrm{C}, A^{-\gamma}\right]$, (resp. by $\left[\mathrm{C}, A_{0}^{-\gamma}\right]$ ), which $\mathrm{C}$ maps continuously into $A^{-\gamma}$ (resp. into $A_{0}^{-\gamma}$ ). More specifically, it is shown in Theorem 3.1 that

$$
\left[C, A^{-\gamma}\right]=\left\{f \in H(\mathbb{D}): f(z) /(1-z) \in A^{-(\gamma+1)}\right\}, \quad \gamma>0,
$$

equipped with the norm $\|f\|=\|f(z) /(1-z)\|_{-(\gamma+1)}$. A similar description is available for $\left[\mathrm{C}, A_{0}^{-\gamma}\right]$; see Theorem 3.2. An important feature is that the containment $A^{-\gamma} \subseteq\left[\mathrm{C}, A^{-\gamma}\right]$ is proper, that is, $\mathrm{C}_{\gamma}: A^{-\gamma} \rightarrow A^{-\gamma}$ has a genuine extension $\mathrm{C}:\left[\mathrm{C}, A^{-\gamma}\right] \rightarrow A^{-\gamma}$ to its optimal domain space, as is the case for $\mathrm{C}_{\gamma, 0}$ acting in $A_{0}^{-\gamma}$. As a consequence, it is shown that the nested family of spaces $A^{-\gamma} \varsubsetneqq A^{-\beta}$ (resp. $A_{0}^{-\gamma} \varsubsetneqq A_{0}^{-\beta}$ ), for $0<\gamma<\beta$, has the property that $\mathrm{C}$ fails to map every larger space $A^{-\beta}$ (resp. $A_{0}^{-\beta}$ ) into $A^{-\gamma}$ (resp. into $A_{0}^{-\gamma}$ ). In particular, $A^{-\beta} \nsubseteq\left[\mathrm{C}, A^{-\gamma}\right]$ and $A_{0}^{-\beta} \nsubseteq\left[\mathrm{C}, A_{0}^{-\gamma}\right]$ for every $\beta>\gamma$. On the other hand, the Banach space of analytic functions [C, $\left.A_{0}^{-\gamma}\right]$ is non-comparable with $A^{-\gamma}$ (i.e., $A^{-\gamma} \nsubseteq\left[\mathrm{C}, A_{0}^{-\gamma}\right]$ and $\left[\mathrm{C}, A_{0}^{-\gamma}\right] \nsubseteq A^{-\gamma}$ ) and, akin to $A^{-\gamma}$, is also a proper subspace of the optimal domain space [C, $\left.A^{-\gamma}\right]$; see Proposition 3.6.

Our notation for concepts from functional analysis and operator theory is standard and we refer to [18, 26, 27], for example. For Banach spaces of analytic functions see [17, 21], for mean ergodic operators [18, 23], and for linear dynamics [10, 19].

\section{Mean ergodic Properties of $\mathrm{C}_{\gamma}$ And $\mathrm{C}_{\gamma, 0}$}

As noted above, $A^{-\gamma}$ is canonically isomorphic to the bidual $\left(A_{0}^{-\gamma}\right)^{* *}$ of the Banach space $A_{0}^{-\gamma}$. In terms of this biduality the operator $\mathrm{C}_{\gamma}: A^{-\gamma} \rightarrow$ $A^{-\gamma}$ is the bidual operator $\left(\mathrm{C}_{\gamma, 0}\right)^{* *}$ of $\mathrm{C}_{\gamma, 0}: A_{0}^{-\gamma} \rightarrow A_{0}^{-\gamma}$. 
We recall the following result of Aleman and Persson (see [28, Theorem 4.1] and [7, Theorems 4.1, 5.1 and Corollaries 2.1, 5.1], that will be used on several occasions.

Theorem 2.1. Let $\gamma>0$. The Cesàro operator $\mathrm{C}_{\gamma, 0}: A_{0}^{-\gamma} \rightarrow A_{0}^{-\gamma}$ has the following properties.

(i) $\sigma_{p t}\left(\mathrm{C}_{\gamma, 0}\right)=\left\{\frac{1}{m}: m \in \mathbb{N}, m<\gamma\right\}$.

(ii) $\sigma\left(\mathrm{C}_{\gamma, 0}\right)=\sigma_{p t}\left(\mathrm{C}_{\gamma, 0}\right) \cup\left\{\lambda \in \mathbb{C}:\left|\lambda-\frac{1}{2 \gamma}\right| \leq \frac{1}{2 \gamma}\right\}$.

(iii) If $\left|\lambda-\frac{1}{2 \gamma}\right|<\frac{1}{2 \gamma}$ (equivalently $\operatorname{Re}\left(\frac{1}{\lambda}\right)>\gamma$ ), then $\operatorname{Im}\left(\lambda I-\mathrm{C}_{\gamma, 0}\right)$ is a closed subspace of $A_{0}^{-\gamma}$ and has codimension 1.

Moreover, the Cesàro operator $\mathrm{C}_{\gamma}: A^{-\gamma} \rightarrow A^{-\gamma}$ satisfies

(iv) $\sigma_{p t}\left(\mathrm{C}_{\gamma}\right)=\left\{\frac{1}{m}: m \in \mathbb{N}, m \leq \gamma\right\}$, and

(v) $\sigma\left(\mathbf{C}_{\gamma}\right)=\sigma\left(\mathbf{C}_{\gamma, 0}\right)$.

As an immediate consequence we have the following result.

Corollary 2.2. For $\gamma>0$, neither of the operators $\mathrm{C}_{\gamma}, \mathrm{C}_{\gamma, 0}$ is weakly compact.

Proof. Fix $\gamma>0$. As noted in Section 1 there exists a Banach space isomorphism $\Phi$ from $c_{0}$ onto $A_{0}^{-\gamma}$. Suppose that $\mathrm{C}_{\gamma, 0}$ is weakly compact. Then $\widetilde{\mathrm{C}}:=\Phi^{-1} \mathrm{C}_{\gamma, 0} \Phi$ is weakly compact from $c_{0}$ into $c_{0}$ and hence, $\widetilde{\mathrm{C}}$ is also compact, [26, Ex. 3.54(b), p.347]. Accordingly, $\mathrm{C}_{\gamma, 0}=\Phi \widetilde{\mathrm{C}} \Phi^{-1}$ is compact. But, this is impossible as $\sigma\left(\mathrm{C}_{\gamma, 0}\right)$ is an uncountable set; see Theorem 2.1(ii). Hence, $\mathrm{C}_{\gamma, 0}$ cannot be weakly compact.

By Gantmacher's Theorem, [18, Ch. VI, Sect. 4, Theorem 8], also $\mathrm{C}_{\gamma}=$ $\left(\mathrm{C}_{\gamma, 0}\right)^{* *}$ cannot be weakly compact.

The continuity of $\mathrm{C}_{\gamma}$ and $\mathrm{C}_{\gamma, 0}$ as established in [7, Theorem 4.1] gives no quantitative estimate for their operator norm. So, the following result is of some interest.

Theorem 2.3. (i) Let $\gamma \geq 1$. Then $\left\|\mathrm{C}_{\gamma}^{n}\right\|=\left\|\mathrm{C}_{\gamma, 0}^{n}\right\|=1$ for all $n \in \mathbb{N}$.

(ii) Let $0<\gamma<1$. Then $\left\|C_{\gamma}^{n}\right\|=\left\|C_{\gamma, 0}^{n}\right\|=1 / \gamma^{n}$ for all $n \in \mathbb{N}$.

Proof. Let $\gamma>0$. Fix $f \in A^{-\gamma}$ with $\|f\|_{\gamma} \leq 1$. Then $|f(\xi)| \leq(1-|\xi|)^{-\gamma}$ for $\xi \in \mathbb{D}$. For $z \in \mathbb{D} \backslash\{0\}$, it follows from (1.1) and the previous inequality for $|f|$ that

$$
\begin{aligned}
\left|\mathrm{C}_{\gamma}(f)(z)\right| & =\frac{1}{|z|}\left|\int_{0}^{1} \frac{f(t z)}{1-t z} z d t\right| \leq \int_{0}^{1} \frac{|f(t z)|}{|1-t z|} d t \leq \int_{0}^{1} \frac{|f(t z)|}{1-t|z|} d t \\
& \leq \int_{0}^{1} \frac{d t}{(1-t|z|)^{\gamma+1}}=\left[\frac{1}{\gamma|z|}(1-t|z|)^{-\gamma}\right]_{t=0}^{t=1} \\
& =\frac{1}{\gamma|z|}\left(\frac{1}{(1-|z|)^{\gamma}}-1\right)=\frac{1}{(1-|z|)^{\gamma}} \frac{1-(1-|z|)^{\gamma}}{\gamma|z|} .
\end{aligned}
$$


The non-negative function $\phi(s):=\frac{1-(1-s)^{\gamma}}{s}$ for $s \in(0,1]$ and $\phi(0):=\gamma$ is continuous as $\lim _{s \rightarrow 0^{+}} \frac{1-(1-s)^{\gamma}}{s}=\gamma$. Set $M_{\gamma}:=\sup _{s \in[0,1]} \phi(s)$. Since $\left|\mathrm{C}_{\gamma}(f)(0)\right|=$ $|f(0)| \leq 1$, we have $1 \leq \sup _{z \in \mathbb{D}}(1-|z|)^{\gamma}\left|C_{\gamma}(f)(z)\right| \leq \max \left\{1, \frac{M_{\gamma}}{\gamma}\right\}$. Hence,

$$
1 \leq\left\|\mathrm{C}_{\gamma}\right\| \leq \max \left\{1, \frac{M_{\gamma}}{\gamma}\right\}, \quad \gamma>0
$$

(i) Let now $\gamma \geq 1$. The claim is that $\frac{M_{\gamma}}{\gamma} \leq 1$, which then implies that $\left\|C_{\gamma}\right\|=1$. To verify the claim it suffices to show that the function $\psi(s):=$ $(1-s)^{\gamma}-1+\gamma s$, for $s \in[0,1]$, is non-negative. Observe that $\psi(0)=0$ and $\psi(1)=(\gamma-1) \geq 0$. If $\gamma=1$, then $\psi(s)=0$ for all $s \in[0,1]$ and we are done. In case $\gamma>1$ we have $\psi^{\prime}(s)=-\gamma(1-s)^{\gamma-1}+\gamma=\gamma\left[1-(1-s)^{\gamma-1}\right] \geq 0$ and so $\psi$ is increasing. In particular, $\psi(s) \geq \psi(0)=0$ for $s \in[0,1]$, as required.

Clearly, $\left\|C_{\gamma}\right\| \leq 1$ implies that $\left\|C_{\gamma}^{n}\right\| \leq\left\|C_{\gamma}\right\|^{n} \leq 1$ for $n \in \mathbb{N}$. On the other hand, Theorem 2.1 shows that $1 \in \sigma\left(C_{\gamma}\right)$ and so, by the spectral mapping theorem, $1 \in \sigma\left(C_{\gamma}^{n}\right)$. In particular, the spectral radius $r\left(C_{\gamma}^{n}\right) \geq 1$, for $n \in \mathbb{N}$, and so $1 \leq r\left(\mathrm{C}_{\gamma}^{n}\right) \leq\left\|\mathrm{C}_{\gamma}^{n}\right\|$ for $n \in \mathbb{N}$. This completes the proof of (i) for $\mathrm{C}_{\gamma}$. The statement for $\mathrm{C}_{\gamma, 0}$ now follows from $\left\|\mathrm{C}_{\gamma, 0}^{n}\right\|=\left\|\left(C_{\gamma, 0}^{n}\right)^{* *}\right\|=\left\|\mathrm{C}_{\gamma}^{n}\right\|$ for $n \in \mathbb{N}$.

(ii) Let $0<\gamma<1$. It follows from Theorem 2.1 (as $\sigma_{p t}\left(C_{\gamma, 0}\right)=\emptyset$ in this case), that $\sigma\left(C_{\gamma, 0}\right)=\left\{\lambda \in \mathbb{C}:\left|\lambda-\frac{1}{2 \gamma}\right| \leq \frac{1}{2 \gamma}\right\}$. In particular, $\left[0, \frac{1}{\gamma}\right] \subseteq$ $\sigma\left(C_{\gamma, 0}\right)$. By the spectral mapping theorem $\left[0, \frac{1}{\gamma^{n}}\right] \subseteq \sigma\left(C_{\gamma, 0}^{n}\right)$ for each $n \in \mathbb{N}$, and so $1 / \gamma^{n} \leq r\left(C_{\gamma, 0}^{n}\right) \leq\left\|C_{\gamma, 0}^{n}\right\|=\left\|C_{\gamma}^{n}\right\|$ for each $n \in \mathbb{N}$.

To establish the reverse inequality we show, because of $0<\gamma<1$, that $M_{\gamma} \leq 1$. Indeed, for $0<s<1$ we have $(1-s)<(1-s)^{\gamma}$, i.e., $1-(1-s)^{\gamma}<s$, and hence, $0<\phi(s)<1$. Since $\phi(0)=\gamma<1$ and $\phi(1)=1$, it is clear that $M_{\gamma} \leq 1$ and so $\frac{M_{\gamma}}{\gamma} \leq \frac{1}{\gamma}$. It follows from (2.1) that $\left\|C_{\gamma}\right\| \leq \frac{1}{\gamma}$. Hence, $\left\|\mathrm{C}_{\gamma}^{n}\right\|=\left\|\mathrm{C}_{\gamma, 0}^{n}\right\| \leq \frac{1}{\gamma^{n}}$ for all $n \in \mathbb{N}$, and the proof is complete.

For a Banach space $X$, denote by $\mathcal{L}(X)$ the space of all bounded linear operators from $X$ into itself. Recall that an operator $T \in \mathcal{L}(X)$ is mean ergodic if its sequence of Cesàro averages

$$
T_{[n]}:=\frac{1}{n} \sum_{m=1}^{n} T^{m}, \quad n \in \mathbb{N},
$$

converges to some operator $P \in \mathcal{L}(X)$ in the strong operator topology $\tau_{s}$, i.e., $\lim _{n \rightarrow \infty} T_{[n]} x=P x$ for each $x \in X$, [18, Ch.VIII]. It follows from (2.2) that $\frac{T^{n}}{n}=T_{[n]}-\frac{n-1}{n} T_{[n-1]}$, for $n \geq 2$. Hence, $\tau_{s}-\lim _{n \rightarrow \infty} \frac{T^{n}}{n}=0$ whenever $T$ is mean ergodic and, in particular, $\sup _{n} \frac{\left\|T^{n}\right\|}{n}<\infty$. According to [18, VIII Corollary 5.2, p.662], when $T$ is mean ergodic one has the direct decomposition

$$
X=\operatorname{Ker}(I-T) \oplus \overline{\operatorname{Im}(I-T)} .
$$


A Banach space operator $T \in \mathcal{L}(X)$ is called uniformly mean ergodic if there exists $P \in \mathcal{L}(X)$ such that $\lim _{n \rightarrow \infty}\left\|T_{[n]}-P\right\|=0$. It is then immediate that necessarily $\lim _{n \rightarrow \infty} \frac{\left\|T^{n}\right\|}{n}=0$. A result of M.Lin, [24], states that a Banach space operator $T \in \mathcal{L}(X)$ satisfying $\lim _{n \rightarrow \infty} \frac{\left\|T^{n}\right\|}{n}=0$ is uniformly mean ergodic if and only if $\operatorname{Im}(I-T)$ is a closed subspace of $X$.

An operator $T \in \mathcal{L}(X)$ is called power bounded if $\sup _{n \in \mathbb{N}}\left\|T^{n}\right\|<\infty$. Since $T_{[n]}(I-T)=\frac{1}{n}\left(T-T^{n+1}\right)$ for $n \in \mathbb{N}$, it follows that

$$
\lim _{n \rightarrow \infty} T_{[n]} x=0, \quad x \in \operatorname{Im}(I-T),
$$

whenever $T$ is power bounded.

Lemma 2.4. (i) Define $\varphi \in H(\mathbb{D})$ via $\varphi(z):=1 /(1-z)$ for $z \in \mathbb{D}$. The Cesàro operator $\mathrm{C}: H(\mathbb{D}) \rightarrow H(\mathbb{D})$ satisfies $\operatorname{Ker}(I-\mathrm{C})=\operatorname{span}\{\varphi\}$ and

$$
\operatorname{Im}(I-\mathrm{C})=\{h \in H(\mathbb{D}): h(0)=0\}=\operatorname{Ker}\left(\delta_{0}\right),
$$

with $\delta_{0} \in H(\mathbb{D})^{*}$. In particular, $\operatorname{Im}(I-C)$ is closed in $H(\mathbb{D})$.

(ii) Let $X$ be any Banach space of analytic functions on $\mathbb{D}$ which contains the constant functions, is continuously included in $H(\mathbb{D})$ and such that $\mathrm{C}$ : $X \rightarrow X$ is continuous. If $\mathrm{C}: X \rightarrow X$ is mean ergodic, then $\varphi \in X$ and $\operatorname{Ker}(I-C)=\operatorname{span}\{\varphi\}$.

Proof. (i) This is proved in [28, Section 2]; see (2.4) and (2.5) on page 1184 with $\lambda=1$.

(ii) Assume that $\mathrm{C}: X \rightarrow X$ is continuous and mean ergodic. Let $\mathrm{C}_{X}$ denote the restriction of $C$ to $X$. Then

$$
X=\operatorname{Ker}\left(I-\mathrm{C}_{X}\right) \oplus \overline{\operatorname{Im}\left(I-\mathrm{C}_{X}\right)} .
$$

If $\varphi \notin X$, then $\operatorname{Ker}\left(I-\mathrm{C}_{X}\right)=\{0\}$ by part (i). Since point evaluations on $\mathbb{D}$ belong to $X^{*}$ (see Section 1), it follows from (2.5) that $X \subseteq\{h \in H(\mathbb{D})$ : $h(0)=0\}$. This is a contradiction, since the constant function $\mathbf{1}$ belongs to $X$. Hence, $\varphi \in X$. Since $\operatorname{Ker}\left(I-C_{X}\right) \subseteq \operatorname{Ker}(I-\mathrm{C})$, it follows from part (i) that $\operatorname{Ker}\left(I-C_{X}\right)=\operatorname{span}\{\varphi\}$.

We can now establish the main result of this section.

Theorem 2.5. (i) Let $0<\gamma<1$. Both of the operators $\mathrm{C}_{\gamma}$ and $\mathrm{C}_{\gamma, 0}$ fail to be power bounded and are not mean ergodic. Moreover,

$$
\operatorname{Ker}\left(I-\mathrm{C}_{\gamma}\right)=\operatorname{Ker}\left(I-\mathrm{C}_{\gamma, 0}\right)=\{0\},
$$

and $\operatorname{Im}\left(I-\mathrm{C}_{\gamma}\right)$ (resp. $\left.\operatorname{Im}\left(I-\mathrm{C}_{\gamma, 0}\right)\right)$ is a proper closed subspace of $A^{-\gamma}$ (resp. of $\left.A_{0}^{-\gamma}\right)$.

(ii) Both of the operators $\mathrm{C}_{1}$ and $\mathrm{C}_{1,0}$ are power bounded but not mean ergodic. Moreover, $\operatorname{Im}\left(I-\mathrm{C}_{1}\right)$ (resp. $\left.\operatorname{Im}\left(I-\mathrm{C}_{1,0}\right)\right)$ is not a closed subspace of $A^{-\gamma}\left(\right.$ resp. of $\left.A_{0}^{-\gamma}\right)$.

(iii) Let $\gamma>1$. Both of the operators $\mathrm{C}_{\gamma}$ and $\mathrm{C}_{\gamma, 0}$ are power bounded and uniformly mean ergodic. Moreover, $\operatorname{Im}\left(I-\mathrm{C}_{\gamma}\right)\left(\right.$ resp. $\left.\operatorname{Im}\left(I-\mathrm{C}_{\gamma, 0}\right)\right)$ is a 
proper closed subspace of $A^{-\gamma}$ (resp. of $\left.A_{0}^{-\gamma}\right)$. In addition,

$$
\operatorname{Im}\left(I-\mathrm{C}_{\gamma}\right)=\left\{h \in A^{-\gamma}: h(0)=0\right\} .
$$

Moreover, with $\varphi(z):=1 /(1-z)$, for $z \in \mathbb{D}$, the linear projection operator $P_{\gamma}: A^{-\gamma} \rightarrow A^{-\gamma}$ given by

$$
P_{\gamma}(f):=f(0) \varphi, \quad f \in A^{-\gamma},
$$

is continuous and satisfies $\lim _{n \rightarrow \infty}\left(\mathrm{C}_{\gamma}\right)_{[n]}=P_{\gamma}$ in the operator norm.

Proof. Clearly the function $\varphi \in A_{0}^{-\gamma} \subseteq A^{-\gamma}$ if $\gamma>1$. Moreover, $\varphi \in$ $A^{-1} \backslash A_{0}^{-1}$ and $\varphi \notin A^{-\gamma}$ in case $0<\gamma<1$.

(i) For $0<\gamma<1$ it is clear from Theorem 2.3(ii) that both $\mathrm{C}_{\gamma}$ and $\mathrm{C}_{\gamma, 0}$ are not power bounded. Neither are they mean ergodic because $\sup _{n}\left\|\mathrm{C}_{\gamma}^{n}\right\| / n=$ $\sup _{n}\left\|\mathrm{C}_{\gamma, 0}^{n}\right\| / n=\infty$; see the discussion after (2.2). Since $\operatorname{Ker}(I-\mathrm{C})=$ $\operatorname{span}\{\varphi\}$ in $H(\mathbb{D})$, by Lemma $2.4(\mathrm{i})$ and because $\varphi \notin A^{-\gamma}$, we can conclude that $\operatorname{Ker}\left(I-\mathrm{C}_{\gamma}\right)=\operatorname{Ker}\left(I-\mathrm{C}_{\gamma, 0}\right)=\{0\}$.

Since $\left|1-\frac{1}{2 \gamma}\right|<\frac{1}{2 \gamma}$, we can apply Theorem 2.1(iii) to deduce that $\operatorname{Im}(I-$ $\left.\mathrm{C}_{\gamma, 0}\right)$ is a proper closed subspace of $A_{0}^{-\gamma}$. Moreover, $\left(I-\mathrm{C}_{\gamma}\right) \in \mathcal{L}\left(A^{-\gamma}\right)$ is the bidual operator of $I-\mathrm{C}_{\gamma, 0}$ and so it follows from [2, Proposition 2.1] that $\operatorname{Im}\left(I-\mathrm{C}_{\gamma}\right)$ is also closed in $A^{-\gamma}$. It is a proper subspace of $A^{-\gamma}$ because every $h \in \operatorname{Im}\left(I-\mathrm{C}_{\gamma}\right)$ satisfies $\mathrm{C}_{\gamma}(h)(0)=0$; see (1.1).

(ii) Both $C_{1}$ and $C_{1,0}$ are power bounded by Theorem 2.3(i). Since $\varphi \notin$ $A_{0}^{-1}$, it follows from Lemma 2.4(ii), with $X=A_{0}^{-1}$, that $\mathrm{C}_{1,0}$ is not mean ergodic in $A_{0}^{-1}$. By [2, Proposition 2.2], $\mathrm{C}_{1}$ also fails to be mean ergodic.

Assume that $\operatorname{Im}\left(I-\mathrm{C}_{1}\right)$ is closed in $A^{-1}$. Since $\lim _{n \rightarrow \infty}\left\|\mathrm{C}_{1}^{n}\right\| / n=0$, it follows from Lin's theorem, [24], that $C_{1}$ is uniformly mean ergodic. But, we just argued that $C_{1}$ is not even mean ergodic; contradiction! The argument for $C_{1,0}$ is the same.

(iii) Let $\gamma>1$. By Theorem 2.3(i) both $\mathrm{C}_{\gamma}$ and $\mathrm{C}_{\gamma, 0}$ are power bounded. In order to verify (2.6), it is clear that $\operatorname{Im}\left(I-\mathrm{C}_{\gamma}\right) \subseteq\left\{h \in A^{-\gamma}: h(0)=0\right\}$.

For the reverse inclusion, fix $h \in A^{-\gamma}$ satisfying $h(0)=0$. Theorem 2.1 reveals that $1 \in \rho\left(\mathrm{C}_{\gamma}\right)$. By the calculations on p.1184 of [28], with $\lambda=1$, there is a unique $f \in H(\mathbb{D})$ satisfying $f(0)=0$ and $(I-C) f=h$. In fact, $f$ is given by [28, formula $(2.7)]$ :

$$
f(z)=h(z)+\frac{1}{1-z} \int_{0}^{z} \frac{h(\zeta)}{\zeta}, \quad z \in \mathbb{D} .
$$

In order to conclude that $f \in A^{-\gamma}$, we first show that the function $H(z):=$ $h(z) / z$, for $z \in \mathbb{D} \backslash\{0\}$ and $H(0):=h^{\prime}(0)$, belongs to $A^{-\gamma}$. Recalling that $h(0)=0$, it is clear that $H \in H(\mathbb{D})$. If $\frac{1}{2} \leq|z|<1$, then $|H(z)| \leq 2|h(z)|$. Hence, $(1-|z|)^{\gamma}|H(z)| \leq 2\|h\|_{-\gamma}$. On the other hand, for $|z| \leq \frac{1}{2}$ we can apply the maximum modulus principle to $H \in H(\mathbb{D})$ to conclude that

$|H(z)| \leq \max _{|\zeta|=1 / 2}|H(\zeta)|=2 \max _{|\zeta|=1 / 2}|h(\zeta)|=2^{\gamma+1} \max _{|\zeta|=1 / 2}(1-|\zeta|)^{\gamma}|h(\zeta)| \leq 2^{\gamma+1}\|h\|_{-\gamma}$.

Summarizing, $\|H\|_{-\gamma}=\sup _{z \in \mathbb{D}}(1-|z|)^{\gamma}|H(z)| \leq 2^{\gamma+1}\|h\|_{-\gamma}$, i.e., $H \in A^{-\gamma}$. 
Consider $G(z):=\int_{0}^{z} H(\zeta) d \zeta$, for $z \in \mathbb{D}$. Clearly $G^{\prime}(z)=H(z)$ for $z \in \mathbb{D}$, with $H \in A^{-\gamma}$. By a classical result of Hardy and Littlewood (consider $p=\infty$ and $\beta:=\gamma$ in [17, Theorem 5.5]), the function $G \in A^{-(\gamma-1)}=A^{-\gamma+1}$ $($ recall $(\gamma-1)>0))$. Therefore, for each $z \in \mathbb{D}$, we have for $\varphi G$ that

$$
\begin{aligned}
& (1-|z|)^{\gamma}|\varphi(z) G(z)|=(1-|z|)^{\gamma}\left|\frac{1}{1-z} \int_{0}^{z} H(\zeta) d \zeta\right| \\
& \leq(1-|z|)^{\gamma-1}\left|\int_{0}^{z} H(\zeta) d \zeta\right| \leq\|G\|_{-\gamma+1}
\end{aligned}
$$

that is, $\varphi G \in A^{-\gamma}$. Hence, also $f=h+\varphi G \in A^{-\gamma}$. This establishes that $\left.\operatorname{Im}\left(I-\mathrm{C}_{\gamma}\right)\right)$ is closed in $A^{-\gamma}$. Since $\mathrm{C}_{\gamma}$ is power bounded, a theorem of Lin [24] implies that $\mathrm{C}_{\gamma}$ is uniformly mean ergodic.

By [2, Proposition 2.1] it follows that also $\left.\operatorname{Im}\left(I-\mathrm{C}_{\gamma, 0}\right)\right)$ is closed in $A_{0}^{-\gamma}$. So, $C_{\gamma, 0}$ is uniformly mean ergodic, again as a consequence of Lin's theorem.

Lemma 2.4(ii), with $X=A^{-\gamma}$, and the mean ergodicity of $\mathrm{C}_{\gamma}$ yield that $\varphi \in A^{-\gamma}$. Since $\delta_{0} \in\left(A^{-\gamma}\right)^{*}$, the formula

$$
P_{\gamma}(f):=f(0) \varphi=\left\langle f, \delta_{0}\right\rangle \varphi, \quad f \in A^{-\gamma},
$$

implies that $P_{\gamma}$ is a continuous projection in $A^{-\gamma}$.

For each $f \in A^{-\gamma}$ we have

$$
\left(\mathrm{C}_{\gamma}\right)_{[n]}(f)=\left(\mathrm{C}_{\gamma}\right)_{[n]}(f-f(0))+f(0)\left(\mathrm{C}_{\gamma}\right)_{[n]}(\mathbf{1}), \quad n \in \mathbb{N} .
$$

On the other hand, $\varphi \in A^{-\gamma}$ satisfies $\left(\mathrm{C}_{\gamma}\right)_{[n]}(\varphi)=\varphi$ for all $n \in \mathbb{N}$; see Lemma 2.4(ii) with $X=A^{-\gamma}$. According to (2.6) the function $h(z):=z \varphi(z)$ for $z \in \mathbb{D}$ belongs to $\operatorname{Im}\left(I-\mathrm{C}_{\gamma}\right)$ and hence, the sequence

$$
\left(\mathrm{C}_{\gamma}\right)_{[n]}(\mathbf{1})=\left(\mathrm{C}_{\gamma}\right)_{[n]}(\varphi-h)=\varphi-\left(\mathrm{C}_{\gamma}\right)_{[n]}(h), \quad n \in \mathbb{N},
$$

converges to $\varphi$ in $A^{-\gamma}$ for $n \rightarrow \infty$; see (2.4). It then follows from (2.7) and the fact that $\lim _{n \rightarrow \infty}\left(\mathrm{C}_{\gamma}\right)_{[n]}(f-f(0))=0$ in $A^{-\gamma}$ via (2.4) (since $(f-f(0)) \in \operatorname{Im}\left(I-\mathrm{C}_{\gamma}\right)$ by $\left.(2.6)\right)$ that

$$
\lim _{n \rightarrow \infty}\left(\mathrm{C}_{\gamma}\right)_{[n]}(f)=f(0) \varphi=P_{\gamma}(f) .
$$

This establishes that $\tau_{s^{-}} \lim _{n \rightarrow \infty}\left(\mathrm{C}_{\gamma}\right)_{[n]}=P_{\gamma}$. But, the uniform mean ergodicity of $\mathrm{C}_{\gamma}$ means that $\left\{\left(\mathrm{C}_{\gamma}\right)_{[n]}\right\}_{n \in \mathbb{N}}$ is a convergent sequence for the operator norm and hence, its operator norm limit must also be $P_{\gamma}$.

Remark 2.6. (i) Let $\gamma \geq 1$. It follows from Theorem 2.1 that the boundary $\partial \mathbb{D}$ of $\mathbb{D}$ satisfies $\sigma\left(\mathbf{C}_{\gamma}\right) \cap \partial \mathbb{D}=\{1\}=\sigma\left(\mathbf{C}_{\gamma, 0}\right) \cap \partial \mathbb{D}$. Since both $\mathbf{C}_{\gamma}$ and $\mathbf{C}_{\gamma, 0}$ are power bounded, it follows from Theorem 1 and the Remark on p.317 of [22] that

$$
\lim _{n \rightarrow \infty}\left\|C_{\gamma}^{n+1}-C_{\gamma}^{n}\right\|=0=\lim _{n \rightarrow \infty}\left\|C_{\gamma, 0}^{n+1}-C_{\gamma, 0}^{n}\right\|
$$

(ii) Let $\gamma>1$. Theorem 2.1 shows that $\lambda=1$ is an isolated singularity of the resolvent map of both $\mathrm{C}_{\gamma}$ and $\mathrm{C}_{\gamma, 0}$. Since both $\mathrm{C}_{\gamma}$ and $\mathrm{C}_{\gamma, 0}$ are uniformly mean ergodic, it follows that 1 is actually a simple pole of the resolvent map, [23, Theorem 2.7, p.90]. 
(iii) For $\gamma \geq 1$ the point $1 \in \sigma\left(\mathbf{C}_{\gamma}\right)=\sigma\left(\mathbf{C}_{\gamma, 0}\right)$ and so the spectral mapping theorem applied to the polynomial $p_{n}(z):=\frac{1}{n} \sum_{m=1}^{n} z^{m}$ (i.e., $\left(\mathrm{C}_{\gamma}\right)_{[n]}=$ $\left.p_{n}\left(\mathrm{C}_{\gamma}\right)\right)$ yields that $1 \in \sigma\left(\left(\mathrm{C}_{\gamma}\right)_{[n]}\right)$, for $n \in \mathbb{N}$. Hence,

$$
1 \leq r\left(\left(\mathrm{C}_{\gamma}\right)_{[n]}\right) \leq\left\|\left(\mathrm{C}_{\gamma}\right)_{[n]}\right\|, \quad n \in \mathbb{N} .
$$

Then Theorem 2.3(i) and the formula $\left(\mathrm{C}_{\gamma}\right)_{[n]}=\frac{1}{n} \sum_{m=1}^{n} \mathrm{C}_{\gamma}^{m}$ imply that

$$
\left\|\left(\mathrm{C}_{\gamma}\right)_{[n]}\right\|=\left\|\left(\mathrm{C}_{\gamma, 0}\right)_{[n]}\right\|=1, \quad n \in \mathbb{N} .
$$

For $0<\gamma<1$ the point $\frac{1}{\gamma} \in \sigma\left(\mathrm{C}_{\gamma}\right)$ and so again the spectral mapping theorem yields that $p_{n}\left(\frac{1}{\gamma}\right)=\frac{1}{n} \sum_{m=1}^{n} \frac{1}{\gamma^{m}} \in \sigma\left(\left(\mathrm{C}_{\gamma}\right)_{[n]}\right)$ for $n \in \mathbb{N}$. In particular,

$$
\frac{1}{n} \sum_{m=1}^{n} \frac{1}{\gamma^{m}} \leq r\left(\left(\mathrm{C}_{\gamma}\right)_{[n]}\right)=r\left(\left(\mathrm{C}_{\gamma, 0}\right)_{[n]}\right), \quad n \in \mathbb{N} .
$$

It then follows from Theorem 2.3(ii) that

$$
\left\|\left(\mathrm{C}_{\gamma, 0}\right)_{[n]}\right\|=\left\|\left(\mathrm{C}_{\gamma}\right)_{[n]}\right\|=\frac{1}{n} \sum_{m=1}^{n} \frac{1}{\gamma^{m}}=\frac{\left(\frac{1}{\gamma^{n}}\right)-1}{(n+1)(1-\gamma)}, \quad n \in \mathbb{N} .
$$

In particular, for $0<\gamma<1$, not only do $\mathrm{C}_{\gamma}$ and $\mathrm{C}_{\gamma, 0}$ fail to be power bounded but, their Cesàro averages $\left\{\left(\mathrm{C}_{\gamma}\right)_{[n]}\right\}_{n=1}^{\infty}$ and $\left\{\left(\mathrm{C}_{\gamma, 0}\right)_{[n]}\right\}_{n=1}^{\infty}$ are also unbounded sequences in $\mathcal{L}\left(A^{-\gamma}\right)$ and $\mathcal{L}\left(A_{0}^{-\gamma}\right)$, respectively.

Concerning the dynamics of $C$ recall that an operator $T \in \mathcal{L}(X)$, with $X$ a separable Fréchet space, is called hypercyclic if there exists $x \in X$ such that the orbit $\left\{T^{n} x: n \in \mathbb{N}_{0}\right\}$ is dense in $X$. If, for some $z \in X$, the projective orbit $\left\{\lambda T^{n} z: \lambda \in \mathbb{C}, n \in \mathbb{N}_{0}\right\}$ is dense in $X$, then $T$ is called supercyclic. Clearly, hypercyclicity implies supercyclicity. It is proved in $[3]$ that $C$ is not supercyclic on $H(\mathbb{D})$. Since the image of a dense subset of $A_{0}^{-\gamma}$ under the natural inclusion map into $H(\mathbb{D})$ is dense in $H(\mathbb{D})$, we have the following consequence.

Proposition 2.7. The Cesàro operator $\mathrm{C}_{\gamma, 0}$ is not supercyclic and hence, also not hypercyclic, in each space $A_{0}^{-\gamma}$, for $\gamma>0$.

\section{Optimal extension of $\mathrm{C}_{\gamma}$ And of $\mathrm{C}_{\gamma, 0}$}

The optimal domain $\left[\mathrm{C}, H^{p}(\mathbb{D})\right]$ of the Cesàro operator $C: H^{p}(\mathbb{D}) \rightarrow$ $H^{p}(\mathbb{D}), 1 \leq p<\infty$, was introduced and thoroughly investigated in [15, Section 3]. The definition given there is rather general and can be applied to $C$ when it acts in any Banach space of analytic functions $X$ on $\mathbb{D}$. Namely,

$$
[\mathrm{C}, X]:=\{f \in H(\mathbb{D}): \mathrm{C}(f) \in X\},
$$

which is a Banach space for the norm

$$
\|f\|_{[\mathrm{C}, X]}:=\|\mathrm{C}(f)\|_{X}, \quad f \in[\mathrm{C}, X],
$$


as of a consequence of $\mathrm{C}: H(\mathbb{D}) \rightarrow H(\mathbb{D})$ being a topological Fréchet space isomorphism. When point evaluations on $\mathbb{D}$ belong to $[\mathrm{C}, X]^{*}$, then $[\mathrm{C}, X]$ is actually a Banach space of analytic functions on $\mathbb{D}$ and $\mathrm{C}$ maps $[\mathrm{C}, X]$ isometrically onto $X$. If, in addition, C acts in $X$, then $X \subseteq[\mathrm{C}, X]$ and the natural inclusion map is continuous. Most important is that $[\mathrm{C}, X]$ is the largest of all Banach spaces of analytic functions $Y$ on $\mathbb{D}$ that $\mathrm{C}$ maps continuously into $X$; the argument is analogous to [15, Remark 3.1]. To describe more concretely which functions from $H(\mathbb{D})$ are members of $[\mathrm{C}, X]$ may not be easy in general: for $X=H^{p}(\mathbb{D})$ this is based on properties of the Littlewood-Paley $g$-function [15, Proposition 3.2 \& Corollary 3.3 ]. To express the norm (3.1) in a more explicit way, if possible, would also be an advantage.

The aim of this section it to investigate the optimal domain spaces $\left[\mathrm{C}, A^{-\gamma}\right]$ and $\left[\mathrm{C}, A_{0}^{-\gamma}\right]$ for $\gamma>0$. Clearly $\left[\mathrm{C}, A_{0}^{-\gamma}\right] \subseteq\left[\mathrm{C}, A^{-\gamma}\right]$. Moreover, with continuous inclusions, we clearly have

$$
A^{-\gamma} \subseteq\left[\mathrm{C}, A^{-\gamma}\right] \varsubsetneqq H(\mathbb{D}) \text { and } A_{0}^{-\gamma} \subseteq\left[\mathrm{C}, A_{0}^{-\gamma}\right] \varsubsetneqq H(\mathbb{D}),
$$

due to the closed graph theorem and the fact that point evaluations on $\mathbb{D}$ belong to $\left[\mathrm{C}, A^{-\gamma}\right]^{*}$ hence, also to $\left[\mathrm{C}, A_{0}^{-\gamma}\right]^{*}$. To verify this latter claim, fix $z_{0} \in \mathbb{D}$ and choose $0<r<1$ with $\left|z_{0}\right|<r$. For $f \in H(\mathbb{D})$ observe that $f(z)=(1-z)(z \mathrm{C}(f)(z))^{\prime}$, for $z \in \mathbb{D}$, which is routine to verify. Given $f \in\left[\mathrm{C}, A^{-\gamma}\right]$, the previous identity and the Cauchy integral formula yield

$$
\begin{gathered}
\left|\left\langle f, \delta_{z_{0}}\right\rangle\right|=\left|f\left(z_{0}\right)\right|=\left|1-z_{0}\right| \cdot\left|\frac{1}{2 \pi i} \int_{|\zeta|=r} \frac{\zeta \mathrm{C}(f)(\zeta)}{\left(\zeta-z_{0}\right)^{2}} d \zeta\right| \leq \\
\frac{\left|1-z_{0}\right| r^{2}}{\left(r-\left|z_{0}\right|\right)^{2}(1-r)^{\gamma}} \sup _{|\zeta|=r}(1-|\zeta|)^{\gamma}|\mathrm{C}(f)(\zeta)| \leq \frac{\left|1-z_{0}\right| r^{2}}{\left(r-\left|z_{0}\right|\right)^{2}(1-r)^{\gamma}}\|\mathrm{C}(f)\|_{-\gamma} .
\end{gathered}
$$

Accordingly, $\left|f\left(z_{0}\right)\right| \leq \frac{\left|1-z_{0}\right| r^{2}}{\left(r-\left|z_{0}\right|\right)^{2}(1-r)^{\gamma}}\|f\|_{\left[\mathrm{C}, A^{-\gamma}\right]}$ and so each evaluation functional $\delta_{z}: f \rightarrow f(z)$ is continuous on both $\left[\mathrm{C}, A^{-\gamma}\right]$ and $\left[\mathrm{C}, A_{0}^{-\gamma}\right]$, for each $z \in \mathbb{D}$.

Theorem 3.1. Let $\gamma>0$ and $\varphi(z):=1 /(1-z)$ for $z \in \mathbb{D}$. The optimal domain $\left[\mathrm{C}, A^{-\gamma}\right]$ of $\mathrm{C}_{\gamma}: A^{-\gamma} \rightarrow A^{-\gamma}$ is isometrically isomorphic to $A^{-\gamma}$ and is given by

$$
\left[\mathrm{C}, A^{-\gamma}\right]=\left\{f \in H(\mathbb{D}): f \varphi \in A^{-(\gamma+1)}\right\} .
$$

Moreover, the norm $\|\cdot\|_{\left[\mathrm{C}, A^{-\gamma}\right]}$ is equivalent to the norm $f \rightarrow\|f \varphi\|_{-(\gamma+1)}$ and the containment $A^{-\gamma} \subseteq\left[\mathrm{C}, A^{-\gamma}\right]$ is proper.

Proof. That $\left[\mathrm{C}, A^{-\gamma}\right]$ is isometrically isomorphic to $A^{-\gamma}$ follows from the facts that $\mathrm{C}:\left[\mathrm{C}, A^{-\gamma}\right] \rightarrow A^{-\gamma}$ is both injective (as $\mathrm{C}$ is bijective on $H(\mathbb{D})$ ) and surjective (by the definition of $\left[\mathrm{C}, A^{-\gamma}\right]$ ), and that it is an isometry (via $(3.1))$.

To verify (3.2) observe, for $f \in H(\mathbb{D})$, that $\mathrm{C}(f) \in A^{-\gamma}$ if and only if the function $z \mapsto \int_{0}^{z} f(\zeta) /(1-\zeta) d \zeta \in A^{-\gamma}$. This follows from the argument in the proof of Theorem 2.5(iii) above, where it is shown that if $h \in A^{-\gamma}$ 
satisfies $h(0)=0$, then also $H(z):=\frac{h(z)}{z} \in A^{-\gamma}$ (the converse is clear from $h(z)=z H(z))$. Note that this part of the argument in the proof of Theorem 2.5(iii) did not require $\gamma>1$ (which was being assumed there). By a result of Hardy and Littlewood, [17, Theorem 5.5], this in turn is equivalent to $f \varphi \in A^{-(\gamma+1)}$.

To verify the equivalence of the norms $\|\cdot\|_{\left[\mathrm{C}, A^{-\gamma}\right]}$ and $f \rightarrow\|f \varphi\|_{-(\gamma+1)}$ we proceed as follows. First, the space $E:=\left\{f \in H(\mathbb{D}): f \varphi \in A^{-(\gamma+1)}\right\}$ endowed with the norm $\|f \varphi\|_{-(\gamma+1)}$, for $f \in E$, is a Banach space which is isomorphic to $A^{-(\gamma+1)}$. On the other hand, since $\mathrm{C}:\left[\mathrm{C}, A^{-\gamma}\right] \rightarrow A^{-\gamma}$ is continuous we have, for some constant $K>0$, that

$$
\|f \varphi\|_{-(\gamma+1)} \leq\|f\|_{-\gamma} \leq K\|f\|_{\left[\mathrm{C}, A^{-\gamma}\right]}, \quad f \in\left[\mathrm{C}, A^{-\gamma}\right] .
$$

Therefore the identity map $I:\left[\mathrm{C}, A^{-\gamma}\right] \rightarrow E$ is a continuous bijection between Banach spaces. By the open mapping theorem its inverse is also continuous. This implies that the two norms are equivalent in $\left[\mathrm{C}, A^{-\gamma}\right]$.

Finally, the function $g(z):=(1-z) /(1+z)^{\gamma+1}$ for $z \in \mathbb{D}$ satisfies $\|g \varphi\|_{-(\gamma+1)} \leq 1$. i.e., $g \in\left[\mathrm{C}, A^{-\gamma}\right]$. However, $g \notin A^{-\gamma}$ since

$$
\sup _{z \in \mathbb{D}} \frac{(1-|z|)^{\gamma}|1-z|}{|1+z|^{\gamma+1}} \geq \sup _{s \in[0,1]} \frac{1+s}{1-s}=\infty \text {. }
$$

This shows that the containment $A^{-\gamma} \subseteq\left[\mathrm{C}, A^{-\gamma}\right]$ is proper.

Theorem 3.2. Let $\gamma>0$ and $\varphi(z):=1 /(1-z)$ for $z \in \mathbb{D}$. The optimal domain $\left[\mathrm{C}, A_{0}^{-\gamma}\right]$ of $\mathrm{C}_{\gamma, 0}: A_{0}^{-\gamma} \rightarrow A_{0}^{-\gamma}$ is isometrically isomorphic to $A_{0}^{-\gamma}$ and is given by

$$
\left[\mathrm{C}, A_{0}^{-\gamma}\right]=\left\{f \in H(\mathbb{D}): f \varphi \in A_{0}^{-(\gamma+1)}\right\} .
$$

Moreover, the norm $\|\cdot\|_{\left[\mathrm{c}, A_{0}^{-\gamma}\right]}$ is equivalent to the norm $f \mapsto\|f \varphi\|_{-(\gamma+1)}$ and the containment $A_{0}^{-\gamma} \subseteq\left[\mathrm{C}, A_{0}^{-\gamma}\right]$ is proper.

Proof. The Banach space $\left[\mathrm{C}, A_{0}^{-\gamma}\right]$ is isometrically isomorphic to $A_{0}^{-\gamma}$; adapt the argument from the beginning of the proof of Theorem 3.1.

To see that $A_{0}^{-\gamma} \subseteq\left[\mathrm{C}, A_{0}^{-\gamma}\right]$ is proper assume, on the contrary, that $A_{0}^{-\gamma}=$ $\left[\mathrm{C}, A_{0}^{-\gamma}\right]$. Then $\mathrm{C}_{\gamma, 0}:\left[\mathrm{C}, A_{0}^{-\gamma}\right]=A_{0}^{-\gamma} \rightarrow A_{0}^{-\gamma}$ is a Banach space isomorphism and so $0 \notin \sigma\left(\mathbf{C}_{\gamma, 0}\right)$; contradiction to Theorem 2.1(ii).

To establish (3.3) is similar to the proof of (3.2) in Theorem 3.1. It is enough to keep in mind the following two facts.

Fact 1. Let $f \in H(\mathbb{D})$ satisfy $f(0)=0$. Then $f \in A_{0}^{-\gamma}$ if and only if $f(z) / z \in A_{0}^{-\gamma}$.

Fact 2. A function $f \in H(\mathbb{D})$ belongs to $A_{0}^{-\gamma}$ if and only if $f^{\prime} \in A_{0}^{-(\gamma+1)}$.

Indeed, by [17, Theorem 5.5] the differentiation operator $D: A^{-\gamma} \rightarrow$ $A^{-(\gamma+1)}$, given by $D(f):=f^{\prime}$, and the integration operator $J: A^{-(\gamma+1)} \rightarrow$ $A^{-\gamma}$, given by $J(f)(z):=\int_{0}^{z} f(\zeta) d \zeta$, for $z \in \mathbb{D}$, are continuous (see also [20, Theorem 2.1(a) \& Proposition 2.2(a)]). Since the space of polynomials $\mathcal{P}$ is dense in $A_{0}^{-\beta}$ for each $\beta>0$ and $\mathcal{P}$ is invariant for both $D$ and $J$, it 
follows that their restrictions $D: A_{0}^{-\gamma} \rightarrow A_{0}^{-(\gamma+1)}$ and $J: A_{0}^{-(\gamma+1)} \rightarrow A_{0}^{-\gamma}$ are continuous. This implies Fact 2.

Now that (3.3) is established, one can argue as in the proof of Theorem 3.1 to show that the stated norms are equivalent.

Remark 3.3. (i) As noted in Section 1, for every $\gamma>0$ the Banach space $A^{-\gamma}$ (resp. $A_{0}^{-\gamma}$ ) is isomorphic to $\ell^{\infty}$ (resp. $c_{0}$ ). In particular, $A^{-\gamma}$ is nonseparable whereas $A_{0}^{-\gamma}$ is separable. Also, $A_{0}^{-\gamma}$ is not weakly sequentially complete and hence, neither is $A^{-\gamma}$ (as it contains $A_{0}^{-\gamma}$ as a closed subspace). Since both $c_{0}$ and $\ell^{\infty}$ fail to have the Radon-Nikodym property, the same is true for $A^{-\gamma}$ and $A_{0}^{-\gamma}$. And so on. Since $A^{-\gamma}$ (resp. $A_{0}^{-\gamma}$ ) is isomorphic to $\left[\mathrm{C}, A^{-\gamma}\right]$ (resp. to $\left[\mathrm{C}, A_{0}^{-\gamma}\right]$ ), we can conclude that the optimal domain spaces $\left[\mathrm{C}, A^{-\gamma}\right]$ and $\left[\mathrm{C}, A_{0}^{-\gamma}\right]$ inherit such properties as those mentioned above (and others) from $A^{-\gamma}$ and $A_{0}^{-\gamma}$, respectively.

(ii) Let $f, g \in H(\mathbb{D})$ satisfy $|g(z)| \leq|f(z)|$ for $z \in \mathbb{D}$. If $f \in A^{-\gamma}$ (resp. $f \in A_{0}^{-\gamma}$ ), for $\gamma>0$, then it is routine to check that also $g \in A^{-\gamma}$ (resp. $g \in A_{0}^{-\gamma}$ ). It follows from (3.2) (resp. (3.3)) that this useful property carries over to the optimal domain space $\left[\mathrm{C}, A^{-\gamma}\right]$ (resp. $\left.\left[\mathrm{C}, A_{0}^{-\gamma}\right]\right)$.

(iii) According to $\left[17\right.$, p.92] there exists $g \in H^{\infty}(\mathbb{D})$ such that $g^{\prime}$ fails to have boundary values at a.e. point in $\partial \mathbb{D}$. Since $H^{\infty}(\mathbb{D}) \subseteq A_{0}^{-\gamma}$, for every $\gamma>0$, and the differentiation operator $D: A_{0}^{-\gamma} \rightarrow A_{0}^{-(\gamma+1)}$ is continuous, it follows that $g^{\prime} \in A_{0}^{-(\gamma+1)}$. Accordingly, for every $\beta>1$ there exists a function in $A_{0}^{-\beta}$ which fails to have a.e. boundary values. Since $A_{0}^{-\beta} \subseteq$ $A^{-\beta} \subseteq\left[\mathrm{C}, A^{-\beta}\right]$ and $A_{0}^{-\beta} \subseteq\left[\mathrm{C}, A_{0}^{-\beta}\right]$ we can conclude, for every $\beta>1$, that there exist functions in the spaces $A_{0}^{-\beta}, A^{-\beta},\left[\mathrm{C}, A_{0}^{-\beta}\right]$ and $\left[\mathrm{C}, A^{-\beta}\right]$ which fail to have a.e. boundary values.

For $\gamma>0$, the following result shows that the largest growth space $A^{-\beta}$ that is contained in $\left[\mathrm{C}, A^{-\gamma}\right]$ is $A^{-\gamma}$. The same is true for $A_{0}^{-\gamma}$ and $\left[\mathrm{C}, A_{0}^{-\gamma}\right]$.

Proposition 3.4. Let $\gamma>0$. For each $\beta>\gamma$, the space $A^{-\beta} \nsubseteq\left[\mathrm{C}, A^{-\gamma}\right]$ and the space $A_{0}^{-\beta} \nsubseteq\left[\mathrm{C}, A_{0}^{-\gamma}\right]$.

Proof. That $A^{-\beta} \nsubseteq\left[\mathrm{C}, A^{-\gamma}\right]$ is a direct consequence of Theorem 3.1 and the fact that the function $f(z):=1 /(1-z)^{\beta} \in A^{-\beta} \backslash\left[\mathrm{C}, A^{-\gamma}\right]$ because $f(z) /(1-z) \notin A^{-(\gamma+1)}$.

Suppose that $A_{0}^{-\beta} \subseteq\left[\mathrm{C}, A_{0}^{-\gamma}\right]$. Then $\mathrm{C}\left(A_{0}^{-\beta}\right) \subseteq A_{0}^{-\gamma}$. Using that $A_{0}^{-\gamma} \subseteq$ $A_{0}^{-\beta}$ continuously and a closed graph argument it follows that the natural inclusion map $\Phi$ for $A_{0}^{-\beta} \subseteq\left[\mathrm{C}, A_{0}^{-\gamma}\right]$ is continuous. Hence, also $\mathrm{C}: A_{0}^{-\beta} \rightarrow$ $A_{0}^{-\gamma}$ is continuous (being the composition of $\Phi$ and $\mathrm{C}:\left[\mathrm{C}, A_{0}^{-\gamma}\right] \rightarrow A_{0}^{-\gamma}$ ). Passing to the bidual yields that $\mathrm{C}: A^{-\beta} \rightarrow A^{-\gamma}$ is continuous, i.e., $A^{-\beta} \subseteq$ $\left[\mathrm{C}, A^{-\gamma}\right]$, which is a contradiction to the previous paragraph. So, $A_{0}^{-\beta} \nsubseteq$ $\left[\mathrm{C}, A_{0}^{-\gamma}\right]$. 
Given $\gamma>0$, Proposition 3.4 raises the question of whether there are any weighted Banach spaces of analytic functions $X$ on $\mathbb{D}$ which satisfy $A^{-\gamma} \subseteq X \subseteq\left[\mathrm{C}, A^{-\gamma}\right]$

A weight $v: \mathbb{D} \rightarrow[0, \infty)$ is called essential if it is radial (i.e., $v(z)=v(|z|)$ for $z \in \mathbb{D})$, continuous, satisfies that $v(r)$ decreases to 0 as $r$ approaches 1 , and there exists a constant $d>0$ such that for each $z_{0} \in \mathbb{D}$ there is $f_{0} \in H(\mathbb{D})$ such that $\left|f_{0}\left(z_{0}\right)\right| \geq d / v\left(z_{0}\right)$ and $v(z)\left|f_{0}(z)\right| \leq 1$ for each $z \in \mathbb{D}$ (i.e., $\left\|f_{0}\right\|_{v} \leq 1$ in the notation of (3.4) below). It is established in [14] that a radial continuous weight $v: \mathbb{D} \rightarrow(0, \infty)$ which is decreasing to 0 in $[0,1)$ as $r$ approaches 1 is essential if and only if there exists a weight $w: \mathbb{D} \rightarrow(0, \infty)$ satisfying the same assumptions as for $v$ such that the function $r \mapsto-\log \left(w\left(e^{r}\right)\right)$ is convex and, for some constant $c>1$, the weight $w$ satisfies

$$
\frac{1}{c} w(r) \leq v(r) \leq c w(r), \quad r \in[0,1) .
$$

In other words, radial continuous weights in $\mathbb{D}$ that are essential are those which are equivalent to "logarithmic convex weights". All the weights $v_{\gamma}(z)=$ $(1-|z|)^{\gamma}$, for $\gamma>0$, considered in this paper are essential. For further examples and properties of essential weights we refer to [12].

Given an essential weight $v$, the weighted space associated with $v$ is defined by

$$
H_{v}^{\infty}:=\left\{f \in H(\mathbb{D})\left|\|f\|_{v}:=\sup _{z \in \mathbb{D}} v(z)\right| f(z) \mid<\infty\right\} ;
$$

it is a Banach space of analytic functions on $\mathbb{D}$ for the norm $\|\cdot\|_{v}$. According to [30, Lemma 1], point evaluations on $\mathbb{D}$ belong to $\left(H_{v}^{\infty}\right)^{*}$.

Proposition 3.5. Let $\gamma>0$ and $v$ be an essential weight on $\mathbb{D}$. If it is the case that $A^{-\gamma} \subseteq H_{v}^{\infty} \subseteq\left[\mathrm{C}, A^{-\gamma}\right]$, then there exists $b>0$ such that

$$
(1 / b)(1-|z|)^{\gamma} \leq v(z) \leq b(1-|z|)^{\gamma}, \quad z \in \mathbb{D} .
$$

In particular, $A^{-\gamma}=H_{v}^{\infty}$, both algebraically and topologically.

Proof. The assumption that $A^{-\gamma} \subseteq H_{v}^{\infty} \subseteq\left[\mathrm{C}, A^{-\gamma}\right]$ and the closed graph theorem imply that both inclusions are continuous. In particular, there is $M>0$ such that $\|f\|_{v} \leq M\|f\|_{-\gamma}$ for each $f \in A^{-\gamma}$ and, by Theorem 3.1, $\|g /(1-z)\|_{-(\gamma+1)} \leq K\|g\|_{v}$ for each $g \in H_{v}^{\infty}($ and some $K>0)$.

Given an arbitrary point $u \in \mathbb{D} \backslash\{0\}$, we set $f_{0}(z):=1 /\left(1-z|u| u^{-1}\right)^{\gamma}$, for $z \in \mathbb{D}$. For $u=0$, define $f_{0}(z)=1$, for $z \in \mathbb{D}$. Then $\left\|f_{0}\right\|_{-\gamma}=1$ and $f_{0}(u)=1 /(1-|u|)^{\gamma}$. Moreover, $v(u)\left|f_{0}(u)\right| \leq\left\|f_{0}\right\|_{v} \leq M$. This implies that $v(u) \leq M(1-|u|)^{\gamma}$ for each $u \in \mathbb{D}$.

Now fix $w \in \mathbb{D}$ arbitrary. Since the weight $v$ is essential, there is $d>0$ (independent of $w$ ) and $g_{0} \in H(\mathbb{D})$ with $\left\|g_{0}\right\|_{v} \leq 1$ (i.e., $g_{0} \in H_{v}^{\infty}$ ) such that $\left|g_{0}(w)\right| \geq d / v(w)$. Observe that

$$
\left\|g_{0} /(1-z)\right\|_{-(\gamma+1)}=\sup _{z \in \mathbb{D}}(1-|z|)^{\gamma+1} \frac{\left|g_{0}(z)\right|}{|1-z|} \leq K\left\|g_{0}\right\|_{v} \leq K .
$$


Accordingly,

$$
\frac{d}{v(w)} \frac{(1-|w|)^{\gamma+1}}{|1-w|} \leq(1-|w|)^{\gamma+1} \frac{\left|g_{0}(w)\right|}{|1-w|} \leq \sup _{z \in \mathbb{D}}(1-|z|)^{\gamma+1} \frac{\left|g_{0}(z)\right|}{|1-z|} \leq K
$$

from which it follows that

$$
\frac{(1-|w|)^{\gamma+1}}{|1-w|} \leq \frac{K}{d} v(w), \quad w \in \mathbb{D} .
$$

In particular, $(1-r)^{\gamma} \leq(K / d) v(r)$ for $r \in[0,1)$. Setting $b:=\max \{M, K / d\}$, yields (3.5).

Proposition 3.6. Let $\gamma>0$.

(i) The containment $A^{-\gamma} \subseteq\left[\mathrm{C}, A^{-\gamma}\right]$ is proper.

(ii) $\left[\mathrm{C}, A_{0}^{-\gamma}\right]$ is a proper, closed subspace of $\left[\mathrm{C}, A^{-\gamma}\right]$.

(iii) The spaces $A^{-\gamma}$ and $\left[\mathrm{C}, A_{0}^{-\gamma}\right]$ are non-comparable. That is,

$$
A^{-\gamma} \nsubseteq\left[\mathrm{C}, A_{0}^{-\gamma}\right] \quad \text { and } \quad\left[\mathrm{C}, A_{0}^{-\gamma}\right] \nsubseteq A^{-\gamma} \text {. }
$$

Proof. (i) Theorem 3.1 shows that $A^{-\gamma}$ is properly contained in [C, $A^{-\gamma}$ ].

(ii) Since $A_{0}^{-\gamma} \subseteq A^{-\gamma}$, it is clear that $\left[\mathrm{C}, A_{0}^{-\gamma}\right] \subseteq\left[\mathrm{C}, A^{-\gamma}\right]$. Moreover, $\left[\mathrm{C}, A_{0}^{-\gamma}\right]$ has the same norm as that in $\left[\mathrm{C}, A^{-\gamma}\right]$; see (3.1). Since $A_{0}^{-\gamma}$ is a closed subspace of $A^{-\gamma}$, it is routine to verify that $\left[\mathrm{C}, A_{0}^{-\gamma}\right]$ is closed in $\left[\mathrm{C}, A^{-\gamma}\right]$. It was noted in the proof of Theorem 3.1 that the function $g(z):=(1-z) /(1+z)^{\gamma+1}$ for $z \in \mathbb{D}$ belongs to $\left[\mathrm{C}, A^{-\gamma}\right]$. However,

$$
(1-|z|)^{\gamma+1}\left|\frac{g(z)}{1-z}\right|=\left(\frac{1-|z|}{|1+z|}\right)^{\gamma+1}, \quad z \in \mathbb{D},
$$

has the value 1 for every real $z \in(-1,0]$ and so, via $(3.3), g \notin\left[\mathrm{C}, A_{0}^{-\gamma}\right]$. Hence, the containment $\left[\mathrm{C}, A_{0}^{-\gamma}\right] \subseteq\left[\mathrm{C}, A^{-\gamma}\right]$ is proper.

(iii) We first show that $A^{-\gamma} \nsubseteq\left[\mathrm{C}, A_{0}^{-\gamma}\right]$. On the contrary, if $A^{-\gamma} \subseteq$ $\left[\mathrm{C}, A_{0}^{-\gamma}\right]$, then it follows that $\mathrm{C}\left(A^{-\gamma}\right) \subseteq A_{0}^{-\gamma}$. Since $\left(A_{0}^{-\gamma}\right)^{* *}=A^{-\gamma}$ and $\left(\mathrm{C}_{\gamma, 0}\right)^{* *}=\mathrm{C}_{\gamma}$, we can conclude that $\left(\mathrm{C}_{\gamma, 0}\right)^{* *}\left(\left(A_{0}^{-\gamma}\right)^{* *}\right) \subseteq A_{0}^{-\gamma}$. But, this contradicts the fact that $\mathrm{C}_{\gamma, 0}$ is not weakly compact; see Corollary 2.2 above and [18, Ch. VI, Sect. 4, Theorem 2]. Accordingly, $A^{-\gamma} \nsubseteq\left[\mathrm{C}, A_{0}^{-\gamma}\right]$.

To show that $\left[\mathrm{C}, A_{0}^{-\gamma}\right] \nsubseteq A^{-\gamma}$ assume, on the contrary, that $\left[\mathrm{C}, A_{0}^{-\gamma}\right] \subseteq$ $A^{-\gamma}$. Via part (i), to achieve a contradiction it suffices to deduce that $\left[\mathrm{C}, A^{-\gamma}\right] \subseteq A^{-\gamma}$.

By the closed graph theorem the inclusion $\left[\mathrm{C}, A_{0}^{-\gamma}\right] \subseteq A^{-\gamma}$ is continuous. Hence, there exists $M>0$ such that

$$
\|h\|_{-\gamma} \leq M\|h\|_{\left[\mathrm{C}, A^{-\gamma}\right]}, \quad h \in\left[\mathrm{C}, A_{0}^{-\gamma}\right],
$$

after noting that the spaces $\left[\mathrm{C}, A_{0}^{-\gamma}\right]$ and $\left[\mathrm{C}, A^{-\gamma}\right]$ have the same norm. Fix $f \in\left[\mathrm{C}, A^{-\gamma}\right]$, in which case $\mathrm{C}(f) \in A^{-\gamma}$. According to Proposition 1.2 of [11] there exists a sequence of polynomials $\left\{p_{j}\right\}_{j \in \mathbb{N}} \subseteq A_{0}^{-\gamma} \subseteq\left[\mathrm{C}, A_{0}^{-\gamma}\right]$ which converges to $\mathrm{C}(f)$ in $H(\mathbb{D})$ and satisfies $\left\|p_{j}\right\|_{-\gamma} \leq\|\mathrm{C}(f)\|_{-\gamma}$ for $j \in \mathbb{N}$. Then $\left\{\mathrm{C}^{-1}\left(p_{j}\right)\right\}_{j \in \mathbb{N}}$ converges to $f$ in $H(\mathbb{D})$ with $\left\{\mathrm{C}^{-1}\left(p_{j}\right)\right\}_{j \in \mathbb{N}} \subseteq\left[\mathrm{C}, A_{0}^{-\gamma}\right] \subseteq A^{-\gamma}$ 
(where the last containment is being assumed). Moreover, $\left\{\mathrm{C}^{-1}\left(p_{j}\right)\right\}_{j \in \mathbb{N}}$ is a bounded set in $A^{-\gamma}$ because

$$
\left\|\mathrm{C}^{-1}\left(p_{j}\right)\right\|_{-\gamma} \leq M\left\|\mathrm{C}^{-1}\left(p_{j}\right)\right\|_{\left[\mathrm{C}, A^{-\gamma}\right]}=M\left\|p_{j}\right\|_{-\gamma} \leq M\|C(f)\|_{-\gamma}, \quad j \in \mathbb{N} .
$$

Since closed balls of $A^{-\gamma}$ are compact sets in the Montel space $H(\mathbb{D})$, it follows that $f \in A^{-\gamma}$. This shows that $\left[\mathrm{C}, A^{-\gamma}\right] \subseteq A^{-\gamma}$ which yields the required contradiction.

Proposition 3.6 shows that the Banach spaces of analytic functions $A^{-\gamma}$ and $\left[\mathrm{C}, A_{0}^{-\gamma}\right]$ are two non-comparable, proper vector subspaces of the optimal domain space $\left[\mathrm{C}, A^{-\gamma}\right]$, that is, $\mathrm{C}$ maps both of these spaces into $A^{-\gamma}$.

Acknowledgements. The research of the first two authors was partially supported by the projects MTM2013-43540-P and GVA Prometeo II $/ 2013 / 013$.

\section{REFERENCES}

[1] A.A. Albanese, J. Bonet, W.J. Ricker, Mean ergodic operators in Fréchet spaces, Ann. Acad. Sci. Fenn. Math. 34 (2009), 401-436.

[2] A.A. Albanese, J. Bonet, W.J. Ricker, Convergence of arithmetic means of operators in Fréchet spaces, J. Math. Anal. Appl. 401 (2013), 160-173.

[3] A.A. Albanese, J. Bonet, W.J. Ricker, The Cesàro operator on power series spaces, Preprint, 2016.

[4] E. Albrecht, T.L. Miller, M.M. Neumann, Spectral properties of generalized Cesàro operators on Hardy and weighted Bergman spaces, Archiv Math. 85 (2005), 446-459.

[5] A. Aleman, A class of integral operators on spaces of analytic functions, in: Proc. of the Winter School in Operator Theory and Complex Analysis, Univ. Málaga Secr. Publ., Málaga, 2007, pp. 3-30.

[6] A. Aleman, O. Constantin, Spectra of integration operators on weighted Bergman spaces, J. Anal. Math. 109 (2009), 199-231.

[7] A. Aleman, A.-M. Persson, Resolvent estimates and decomposable extensions of generalized Cesàro operators, J. Funct. Anal. 258 (2010), 67-98.

[8] A. Aleman, A.G. Siskakis, An integral operator on $H^{p}$, Complex Var. Theory Appl. 28 (1995), 149-158.

[9] A. Aleman, A.G. Siskakis, Integration operators on Bergman spaces, Indiana Univ. Math. J. 46 (1997), 337-356.

[10] F. Bayart, E. Matheron, Dynamics of Linear Operators, Cambridge Univ. Press, Cambridge, 2009.

[11] K.D. Bierstedt, J. Bonet, A. Galbis, Weighted spaces of holomorphic functions on balanced domains, Michigan Math. J. 40 (1993), 271-297.

[12] K.D. Bierstedt, J. Bonet, J. Taskinen, Associated weights and spaces of holomorphic functions, Studia Math. 127 (1998), 137-168.

[13] K.D. Bierstedt, W. H. Summers, Biduals of weighted Banach spaces of analytic functions, J. Austral. Math. Soc. 54 (1993), 70-79.

[14] J. Bonet, P. Domanski, M. Lindström, Essential norm and weak compactness on weighted Banach spaces of analytic functions, Canad. Math. Bull. 42 (1999), 139148.

[15] G.P. Curbera, W.J. Ricker, Extensions of the classical Cesàro operator on Hardy spaces, Math. Scand. 108 (2011), 279-290. 
[16] N. Danikas, A. Siskakis, The Cesàro operator on bounded analytic functions, Analysis 13 (1993), 295-299.

[17] P. Duren, Theory of $H^{p}$ Spaces, Academic Press, New York, 1970.

[18] N. Dunford, J.T. Schwartz, Linear Operators I: General Theory. 2nd Printing, Wiley Interscience Publ., New York, 1964.

[19] K. Grosse-Erdmann, A. Peris, Linear Chaos, Springer Verlag, London, 2011.

[20] A. Harutyunyan, W. Lusky, On the boundedness of the differentiation operator between weighted spaces of holomorphic functions, Studia Math. 184 (2008), 233-247.

[21] H. Hedenmalm, B. Korenblum, K. Zhu, Theory of Bergman Spaces. Grad. Texts in Math. 199, Springer-Verlag, New York, 2000.

[22] Y. Katzelson, L. Tzafriri, On power bounded operators, J. Funct. Anal. 68 (1968), $313-328$.

[23] U. Krengel, Ergodic Theorems. de Gruyter Studies in Mathematics, 6. Walter de Gruyter Co., Berlin, 1985.

[24] M. Lin, On the uniform ergodic theorem. Proc. Amer. Math. Soc. 43 (1974), 337340.

[25] W. Lusky, On the isomorphism classes of weighted spaces of harmonic and holomorphic functions, Studia Math. 175 (1) (2006), 19-40.

[26] R.E. Megginson, An Introduction to Banach Space Theory. Springer, New York Berlin Heidelberg, 1998.

[27] R. Meise, D. Vogt, Introduction to Functional Analysis. Clarendon Press, Oxford, 1997.

[28] A.-M. Persson, On the spectrum of the Cesàro operator on spaces of analytic functions. J. Math. Anal Appl. 340 (2008), 1180-1203.

[29] L.A. Rubel, A.L. Shields, The second dual of certain spaces of analytic functions, J. Austral. Math. Soc. 11 (1970), 276-280.

[30] A.L. Shields, D.L. Williams, Bounded projections, duality and multipliers in spaces of analytic functions, Trans. Amer. Math. Soc. 162 (1971), 287-302.

[31] A. Siskakis, Volterra operators on spaces of analytic functions - a survey, in: Proc. of the First Advanced Course in Operator Theory and Complex Analysis, Univ. Sevilla Serc. Publ., Seville, 2006, pp. 51-68.

Angela A. Albanese, Dipartimento di Matematica e Fisica "E. De Giorgi", Università del Salento- C.P.193, I-73100 Lecce, Italy

E-mail address: angela.albanese@unisalento.it

José Bonet, Instituto Universitario de Matemática Pura y Aplicada

iUmpa, Universitat Politècnica de València, E-46071 Valencia, Spain

E-mail address: jbonet@mat.upv.es

Werner J. Ricker, Math.-Geogr. Fakultät, Katholische Universität Eichstätt-

IngOLSTADT, D-85072 EichstätT, Germany

E-mail address: werner.ricker@ku.de 